\title{
Dome Fuji Seeing -the Summer Results and the Future Winter-over Observations
}

\author{
Hirofumi Okita ${ }^{1}$, Naruhisa Takato ${ }^{2}$, Takashi Ichikawa ${ }^{1}$, \\ Colin S. Bonner ${ }^{3}$, Michel C. B. Ashley ${ }^{3}$, John W. V. Storey ${ }^{3}$ \\ and the $\mathbf{5 1}^{\text {st }}$ and $\mathbf{5 2}^{\text {nd }}$ JARE Dome Fuji teams \\ ${ }^{1}$ Astronomical Institute, Tohoku University, \\ 6-3 Aramaki, Aoba-ku, Sendai, Japan \\ email: h-okita@astr.tohoku.ac.jp \\ ${ }^{2}$ Subaru Telescope, National Astronomical Observatory of Japan, \\ 650 North A'ohoku Place, Hilo, Hawaii \\ ${ }^{3}$ School of Physics, University of New South Wales, \\ NSW 2052, Australia
}

\begin{abstract}
We carried out the first seeing measurements at Dome Fuji in the 2010-2011 austral summer. From these observations, we found that the summer seeing at Dome Fuji was $1.2^{\prime \prime}$ (mean), 1.1" (median), $0.83^{\prime \prime}$ (25th percentile) and $1.5^{\prime \prime}$ (75th percentile), respectively. We also found that the seeing changed continuously and had a minimum around $0.7^{\prime \prime}$ at $\sim 18: 00$ hours daily. We compared the seeing with some weather parameters obtained from the $16 \mathrm{~m}$ mast, and found that the seeing had good correlations with atmosphere temperature and wind shear. These results suggest that the seeing is degraded by turbulence near the surface boundary layer. Because the data were obtained only over a short duration in summer, the general characteristics of Dome Fuji's seeing could not be evaluated. We plan to observe the seeing in winter with a stand-alone DIMM telescope. This new DIMM, which we named the Dome Fuji Differential Image Motion Monitor (DF-DIMM), will be installed at Dome Fuji in January 2013.
\end{abstract}

Keywords. site testing, atmospheric effects, telescopes

\section{Introduction}

The Antarctic plateau is considered as the last frontier for ground-based astronomy. The extremely dry atmosphere, which is related to its low temperature and high altitude of the Antarctic plateau, provides high atmospheric transmittance. To confirm this, site-testing has been carried out at several Antarctic plateau sites (Peterson et al. 2003, Tomasi et al. 2008, Ishii et al. 2010, Sims et al. 2012). Furthermore, this sitetesting showed extremely good seeing on the Antarctic plateau (Loewenstein et al. 1998, Travouillon et al. 2003a, 2003b, Aristidi et al. 2003, 2005, 2009, Lawrence et al. 2004, Bonner et al. 2010). Seeing is a parameter that describes how blurry a star image will be. Poor seeing decreases spatial resolution and detection limits. It is important to carry out astronomical observations at good seeing sites. Table 1 shows the measured seeing and the height of the surface boundary layers at various sites on the Antarctic plateau.

Dome Fuji is located at $77^{\circ} 19^{\prime} \mathrm{S}, 39^{\circ} 42^{\prime} \mathrm{E}$. The altitude of Dome Fuji is about $3,810 \mathrm{~m}$, which is a local maxima, the second highest next to Dome A. Some simulations predict that the seeing and the height of the boundary layer at Dome Fuji is even better than at Dome A or at Dome C (Swain \& Gallée 2006, Saunders et al. 2009). The first seeing measurements at Dome Fuji were carried out in the 2010-2011 austral summer by the $51^{\text {st }}$ and $52^{\text {nd }}$ Japan Antarctic Research Expedition (JARE). 
Table 1. Seeing and the height of the surface boundary layer on the Antarctic plateau.

\begin{tabular}{lccc}
\hline Site & Elevation & $\begin{array}{c}\text { Free atmosphere } \\
\text { seeing }\end{array}$ & $\begin{array}{c}\text { Height of the surface } \\
\text { boundary layer }\end{array}$ \\
\hline South Pole $^{1}$ & $2,835 \mathrm{~m}$ & 0.37 arcsec & $270 \mathrm{~m}$ \\
Dome A $^{2}$ & $4,093 \mathrm{~m}$ & - & $13.9 \mathrm{~m}$ \\
Dome C $^{3}$ & $3,250 \mathrm{~m}$ & 0.36 arcsec & $23 \sim 27 \mathrm{~m}$ \\
Dome Fuji & $3,810 \mathrm{~m}$ & - & - \\
\hline
\end{tabular}

Notes: ${ }^{1}$ Travouillon et al. (2003a); ${ }^{2}$ Bonner et al. (2010); ${ }^{3}$ Aristidi et al. (2009).

\section{Summer seeing in 2010-2011}

We carried out the first seeing measurements at Dome Fuji from January 25 to January 28,2011 . We used a $40 \mathrm{~cm}$ primary mirror telescope for Differential Image Motion Monitor (DIMM) observations at Dome Fuji. DIMMs are now widely used for seeing measurements. This telescope was installed on the snow surface, and the DIMM entrance pupils were about $2 \mathrm{~m}$ above snow surface.

Figure 1 is the time series data of the DIMM seeing at Dome Fuji. The mean seeing was $1.2^{\prime \prime}$, and the median was $1.1^{\prime \prime}$, the 25 th percentile and the 75 th percentile were $0.83^{\prime \prime}$ and $1.5^{\prime \prime}$, respectively.

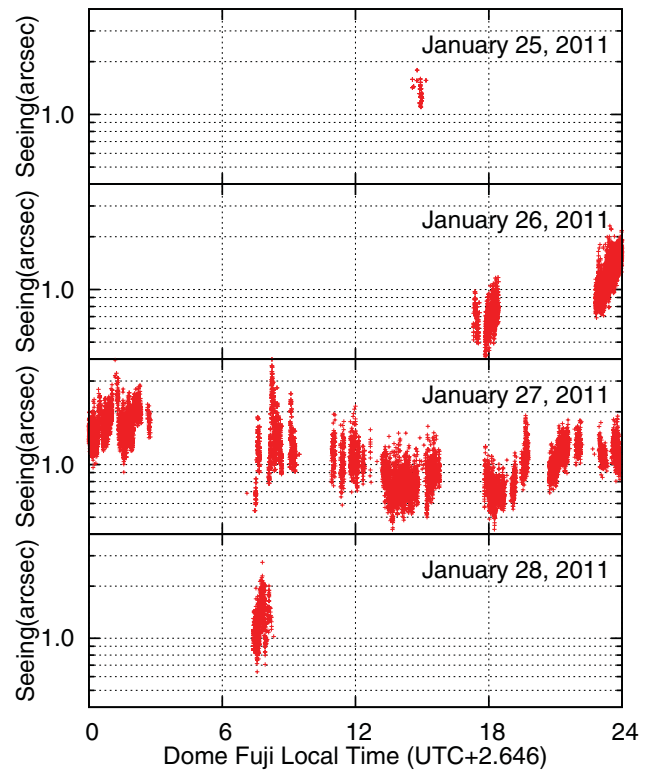

Figure 1. DIMM seeing at the wavelength $\lambda=0.55 \mu \mathrm{m}$ from January 25 to January 28,2011 as a function of the Dome Fuji local time, UTC+2.646. All measurements were carried out during daytime (when the Sun does not set). We chose exposure times of $1 / 1,000$ second. The height of the entrance pupils of the DIMM apertures was $\sim 2 \mathrm{~m}$ above the snow surface.

We examined the time dependence of the seeing. Figure 2 (i) is the seeing averaged in one-hour intervals. The seeing changed continuously and had a minimum around $0.7^{\prime \prime}$ at $\sim 18: 00$ hours. This trend is similar to the day-time seeing at Dome $\mathrm{C}$ (Aristidi et al. 2005). From comparison with the seeing and some meteorological data measured by the $16 \mathrm{~m}$ weather mast, we found that the seeing time dependence correlates with the atmospheric temperature and the wind shear near the snow surface. Figure 2 (ii) and (iii) show these results, averaged for each one-hour observation for the temperature $\left({ }^{\circ} \mathrm{C}\right)$ and wind speed $(\mathrm{m} / \mathrm{s})$. 


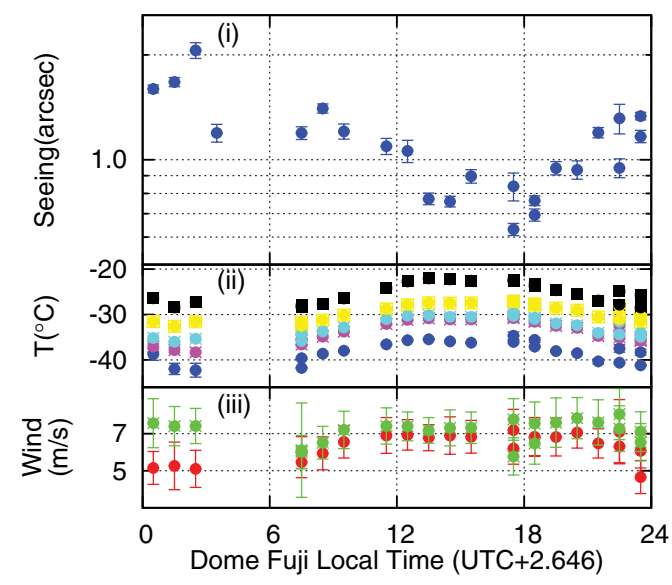

Figure 2. (i) Average seeing in one-hour intervals versus Dome Fuji local time for all seeing data in the 2010-2011 campaign. The error bars indicated the standard error. (ii) and (iii) are the one-hour averaged data measured at the $16 \mathrm{~m}$ weather mast; (ii) temperature with arbitrary offsets, (iii) wind speed. In (ii), the blue, magenta, cyan, yellow and black dots represent the temperature at $0.3 \mathrm{~m}$ above snow surface with offset $-6^{\circ} \mathrm{C}, 6.5 \mathrm{~m}$ with offset $-3^{\circ} \mathrm{C}, 9.5 \mathrm{~m}$ without offset, $12 \mathrm{~m}$ with offset $+3^{\circ} \mathrm{C}$, and $15.8 \mathrm{~m}$ with offset $+6^{\circ} \mathrm{C}$, respectively. The red and the green dots in (iii) are the results at height of $6.1 \mathrm{~m}$ and $14.4 \mathrm{~m}$. The error bar for the meteorological data indicates the standard deviation of each one-hour interval.

Figure 3 (left) is a scatter-gram showing the seeing with atmospheric temperature near the snow surface. The correlation coefficient between the seeing and the temperature is $-0.91 \sim-0.74$. This correlation suggests that the seeing is sensitive to the temperature variation near the snow surface, or the surface boundary layer. The scatter-gram of the seeing and the wind shear, which are measured at heights of $6.1 \mathrm{~m}$ and $14.4 \mathrm{~m}$, are plotted in Figure 3 (right). The correlation coefficient is 0.81 . The friction inside the atmosphere is caused by the wind shear, resulting in turbulence. Hence the seeing would change due to variations in the turbulence strength near the snow surface.
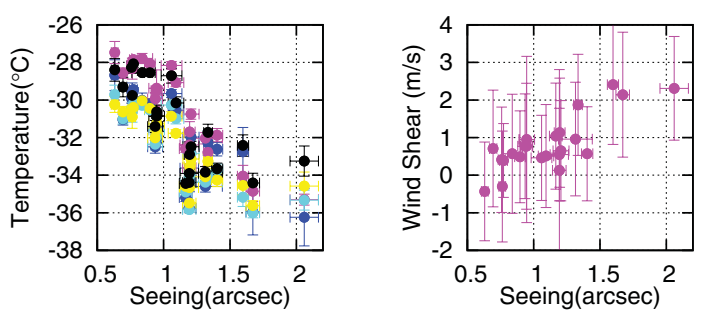

Figure 3. (Left) Correlation between seeing and surface temperature. The abscissa is seeing in arcsec, and the ordinate is the temperature averaged over each one-hour observation. (Right) Correlation between seeing and wind shear.

The general characteristics of Dome Fuji's seeing could not be evaluated from our results because the data were obtained over only a short duration in summer. As there is no sunrise in the Antarctic winter (i.e. polar night), the conditions of the atmosphere at Dome Fuji will be not then be the same as in summer. 


\section{Future seeing measurements}

We aim to determine the winter-time seeing at Dome Fuji. In the 2012-2013 campaign there was no winter crew at Dome Fuji station, so we have to operate any instruments automatically. PLATO-F (Hengst et al. 2010; Ashley et al. 2010), which was installed at Dome Fuji in January 2011, will be the provide for this unmanned operation. It supplies a maximum of $2 \mathrm{~kW}$ of electric power and Iridium internet communications.

We developed a stand-alone DIMM telescope. We call this new telescope the "Dome Fuji Differential Image Motion Monitor (DF-DIMM)". We used a Meade LX200-8" ACF for the telescope and SBIG ST-i CCD cameras for detector and wide-field finder. We replaced the grease, bearings and cables. We placed some heaters inside for $-80^{\circ} \mathrm{C}$ operation. The $\mathrm{C}$ language, awk and bash scripts were used for DF-DIMM software. Pointing, focusing, seeing measurements and data transfer are carried out automatically. We plant to set up the DF-DIMM this austral summer, and then will start annual seeing measurements at Dome Fuji from 2013.

\section{Acknowledgements}

We acknowledge the National Institute of Polar Research, Japan for funding and for the logistics support. Kentaro Motohara gave us his user-friendly DIMM software and some technical support. This work has been supported in part by a Grant-in-Aid for Scientific Research 18340050 and 21244012 of the Ministry of Education, Culture, Sports, Science and Technology in Japan. Hirofumi Okita thanks the scholarships and grant-in-aid of Tohoku University International Advanced Research and Education Organization.

\section{References}

Aristidi, E., Agabi, A., Vernin, J., et al. 2003, A\&GA, 406, L19

Aristidi, E., Agabi, A., Fossat, E., et al. 2005, A\&A, 444, 651

Aristidi, E., Fossat, E., Agabi, A., et al. 2009, A\& A, 499, 955

Ashley, M. C. B., Bonner, C. S., Everett, J. R., et al. 2010, Proc. SPIE, 7735, 133

Bonner, C. S., Ashley, M. C. B., Cui, X., et al. 2010, PASP, 122, 1122

Hengst, S., Luong-Van, D. M., Everett, J. R., et al. 2010, International Journal of Energy Research, 34, 827

Ishii, S., Seta, M., Nakai, N., et al. 2010, Polar Science, 3, 213

Lawrence, J. S., Ashley, M. C. B., Tokovinin, A., \& Travouillon, T. 2004, Nature, 431, 278

Loewenstein, R. F., Bero, C., Lloyd, J. P., et al. 1998, ASP Conf. Ser., 141, 296

Peterson, J. B., Radford, S. J. E., Ade, P. A. R., et al. 2003. PASP, 115, 383

Saunders, W., Lawrence, J. S., Storey, J. W. V., et al. 2009, PASP, 121, 976

Sims, G, Ashley, M. C. B., Cui, X., et al. 2012, PASP, 124, 74

Swain, M. \& Gallée, H. 2006, PASP, 118, 1190

Tomasi, C., Petkov, B., Benedetti, E., et al. 2008 J Atmos Ocean Technol, 25, 213

Travouillon, T., Ashley, M. C. B., Burton, M. G., Storey, J. W. V., \& Loewenstein, R. F. 2003, $A \mathscr{E} A, 400,1163$

Travouillon, T., Ashley, M. C. B., Burton, et al. 2003, A\&A, 409, 1169 